\title{
Estudo do Potencial do PACU (Piaractus mesopotamicus) COMO AGENTE DE CoNTROLE BIOLÓGICo DE Egeria densa, E. najas E Ceratophyllum demersum ${ }^{1}$
}

\author{
Evaluation of the Biocontrol Potential of Pacu (Piaractus mesopotamicus) for Egeria densa, \\ E. najas and Ceratophyllum demersum
}

MIYAZAKI, D.M.Y. ${ }^{2}$ e PITELLI, R.A. ${ }^{3}$

\begin{abstract}
RESUMO - Visando fornecer subsídios para elaboração de sistema de manejo integrado das grandes massas de plantas daninhas aquáticas submersas em lagos e represas, o presente trabalho teve como objetivo verificar a eficiência do pacu (Piaractus mesopotamicus) como agente de controle biológico de Egeria densa, E. najas e Ceratophyllum demersum. As espécies de plantas daninhas foram oferecidas individualmente, duas a duas e as três espécies juntas. Verificou-se que este peixe tem uma eficiência média de controle dessas plantas daninhas variando entre 28 e $100 \%$, podendo eliminar uma massa verde dessas plantas, com a mesma quantidade de seu peso, em sete dias. A eficiência de controle diária aumentou com o tempo de predação. O pacu é mais seletivo para E. densa ou E. najas quando na presença de C. demersum. Não ocorreu alteração na eficiência de controle do pacu sobre $E$. densa ou E. najas em todos os tratamentos e nos três períodos estudados (três, cinco e sete dias).
\end{abstract}

Palavras-chave: plantas daninhas aquáticas, peixes.

\begin{abstract}
In order to provide basic information for the establishment of integrated management systems for submersed aquatic weeds in lakes and reservoirs, this research evaluated the efficiency of pacu (Piaractus mesopotamicus) as a biocontrol agent of Egeria densa, E. najas and Ceratophyllum demersum. One, two and the three plant species together were offered to the fish. The average efficacy of this fish to control these plants ranged between 28 and $100 \%$. The fish could eliminate a fresh mass of these plants, equivalent to its body weight, in seven days of predation period. Control efficiency increased with increasing duration of the trials. Pacu is more selective in controlling $\mathbf{E}$. densa or E. najas when in the presence of C. demersum. No decreased efficacy was observed in the control of E. densa or E. najas in any of the treatments nor in any of three time periods studied (3, 5, and 7 days).
\end{abstract}

Key words: aquatic weeds, fish.

\section{INTRODUÇÃO}

As macrófitas aquáticas são importantes componentes de lagos, rios, reservatórios e outras coleções d'água, pois constituem significativa parcela do estoque de energia e matéria do primeiro nível trófico da rede alimentar, além de proporcionar abrigo para desova e proteção das fases jovens de organismos aquáticos, promovendo heterogeneidade espacial, que favorece a maior biodiversidade local, entre outros efeitos desejáveis (Boyd, 1971; Barko et al., 1986). No entanto, devido à alteração do ambiente aquático promovida pela ação antrópica, algumas espécies são favorecidas e passam a desenvolver densas infestações, promovendo uma série de prejuízos ao equilíbrio biológico do sistema e às atividades do homem,

Recebido para publicação em 6.9.2003 e na forma revisada em 6.10.2003.

2 Eng.-Agr., Aluno do Programa de Pós-Graduação da Produção Vegetal em Agronomia, Faculdade de Ciências Agrárias e Veterinárias, UNESP, 14870-000 Jaboticabal-SP; ${ }^{3}$ Professor Titular, Faculdade de Ciências Agrárias e Veterinárias, UNESP. 
sendo consideradas plantas daninhas e passando, assim, a ser alvo de controle.

Várias são as estratégias de controle de plantas daninhas aquáticas, incluindo o controle físico ou mecânico (sombreamento, drenagem, mecanização e controle manual em ambientes pequenos, como os tanques de piscicultura), o químico (à base de produtos com ação herbicida, como sulfato de cobre, dichlobenil, diquat, diuron, endothal, 2,4-D, fenoprop, glyphosate e e triazina) (Rimer, 1984) e o biológico, utilizando carpa-capim, peixe-boi, patos, gansos, insetos, ácaros, caramujos, plantas competidoras e fungos (Blackburn et al., 1971; Haller, 1976; Sutton \& Vandiver, 1986; Bua-Ngam \& Mercado, 1976).

Recentemente, Nachtigal (2000) isolou e desenvolveu o fungo Fusarium graminearum (isolado FCAV\#940) para o controle biológico de Egeria densa e E. najas, demonstrando grande perspectiva de utilização como bio-herbicida. Entretanto, alguns agentes de controle apresentam restrições à sua utilização, como é o caso da carpa-capim (Ctenopharyngodon idella), que, apesar de excelente predador de plantas daninhas aquáticas, é uma espécie exótica que se reproduz facilmente em novos ambientes (Stanley, 1974), podendo competir diretamente com as espécies nativas.

Os estudos de Da Silva (1982) revelam o potencial do pacu (Piaractus mesopotamicus) como um agente de controle biológico de plantas daninhas aquáticas, pois, dos itens alimentares encontrados no conteúdo estomacal desse peixe, em ambiente natural, a porção vegetal constituiu até $81,9 \%$ em freqüência e $92,1 \%$ em volume; folhas e caules representam até $50,7 \%$ em volume e $32,9 \%$ em freqüência. O pacu destaca-se, também, pelo fato de ser uma espécie nativa e de fácil reprodução e manejo em cativeiro (Castagnolli, 1992), além de controlar plantas daninhas aquáticas em tanques de piscicultura.

Somando a esses fatos a preocupação com a necessidade de informações básicas para implantação de um manejo integrado das grandes massas de plantas daninhas submersas existentes no Brasil, o presente trabalho teve como objetivo verificar a eficiência do pacu (Piaractus mesopotamicus) como agente de controle biológico das plantas submersas Egeria densa, E. najas e Ceratophyllum demersum.

\section{MATERIAL E MÉTODOS}

Os experimentos foram conduzidos no Laboratório Giorgio de Marinis do Dep. de Biologia Aplicada da Faculdade de Ciências Agrárias e Veterinárias, Unesp, campus de Jaboticabal - SP.

Foram utilizados 42 tanques circulares de concreto armado - com 1,20 m de diâmetro, $0,61 \mathrm{~m}$ de altura e volume de 570 litros - com uma taxa de renovação da água de 3,5 vezes ao dia, protegidos por armações com telas do tipo "sombrite" com 50\% de sombreamento, para evitar a instalação de populações de insetos vetores de doenças.

Utilizaram-se juvenis de pacu (Piaractus mesopotamicus), obtidos no Centro de Aqüicultura da UNESP de Jaboticabal, com peso variando entre 80 e 200 g e comprimento de 12 a $16 \mathrm{~cm}$, oriundos de desovas induzidas dentro da mesma semana.

Após a coleta, os peixes foram aclimatados nos tanques durante duas semanas, período no qual foram alimentados duas vezes ao dia com ração balanceada comercial (com 12\% de umidade máxima, $32 \%$ de proteína bruta, $7 \%$ de extrato etério, $9 \%$ de matéria fibrosa e $12 \%$ de matéria mineral) e tratados com sal comum $(\mathrm{NaCl}-0,5 \%)$ por três dias, visando diminuir o estresse causado pelo manejo de captura e transporte. Os peixes foram considerados aptos à experimentação quando voltaram a se alimentar, respeitando-se sempre as duas semanas de aclimatação. Foram oferecidas também pequenas porções de Egeria densa, E. najas e de Ceratophyllum demersum, durante o período de aclimatação.

Em observações prévias, constatou-se que, nos tanques com relação de biomassa entre peixe e planta de 1:1, elas foram controladas por completo entre cinco e oito dias de exposição. Observou-se também que as plantas foram controladas mais rapidamente quando oferecidas soltas na superfície do que quando agrupadas em maços, submersos ou não. Os peixes foram ativos somente durante o dia, não se observando peixes predando plantas durante o periodo noturno. Assim, optou-se por colocar as plantas nas parcelas pela manhã, com períodos de duração dos experimentos de três, cinco e sete dias, sendo o primeiro repetido. 
A eficiência de controle foi avaliada pela fórmula adaptada por Henderson e Tilton, citados por Gravena \& Lara (1976), assim apresentada:

$$
\% E f .=\left(1-\frac{T a \times T r d}{T d \times T r a}\right) \times 100
$$

em que

$\% E f$. = eficiência de controle em porcentagem;

$T a=$ biomassa de plantas encontradas nos tratamentos sem peixes no início do ensaio;

$T d=$ biomassa de plantas encontradas nos tratamentos sem peixes no final do ensaio;

Tra $=$ biomassa de plantas encontradas nos tratamentos com peixes no início do ensaio; e

Trd $=$ biomassa de plantas encontradas nos tratamentos com peixes no final do ensaio.

Também foi calculada a taxa de controle diário, dividindo-se o valor da eficiência de controle pelo tempo de duração do experimento, a qual expressa quanto o peixe controla por dia em relação ao seu peso em porcentagem.

Os tratamentos foram constituídos por dois grupos complementares, com e sem a presença do pacu (agente de controle), necessários para cálculo da eficiência de controle. As plantas foram oferecidas individualmente, duas a duas e as três espécies juntas. Todos os tratamentos possuiam três repetições, totalizando 14 tratamentos e 42 parcelas.

As parcelas dos tratamentos com peixes abrigavam 10 animais, com biomassa total de aproximadamente $1,0 \mathrm{~kg}$ e mesma quantidade de biomassa de plantas, ou seja, com uma relação entre o agente de controle e a planta-alvo fixa de $1: 1$, em biomassa. As parcelas dos tratamentos sem peixes foram constituídas por apenas $1,0 \mathrm{~kg}$ de biomassa de planta. Nos tratamentos em que as espécies de plantas foram colocadas duas a duas ou as três espécies juntas, a biomassa de planta total foi dividida em partes iguais, respeitando-se a biomassa total de $1,0 \mathrm{~kg}$ por parcela.
Realizou-se biometria dos peixes antes e depois de cada experimento, em que se avaliaram o peso e o comprimento, este último tomado, em ictiômetro, partindo do início da boca até a última escama da nadadeira caudal. Para a realização das biometrias os peixes foram deixados em jejum durante um dia e, em seguida, anestesiados em solução de benzocaina $0,1 \mathrm{~g} \mathrm{~L}^{-1}$, por alguns segundos.

As plantas utilizadas foram coletadas no Reservatório de Jupiá, CESP, nas imediações do município de Itapura-SP. Apenas foram utilizados os "ponteiros" das plantas, com comprimento variando de 10 a $80 \mathrm{~cm}$.

As plantas foram colocadas nas parcelas um dia após a biometria dos peixes e retiradas logo após o período de controle. Para determinação do peso da biomassa das plantas, estas foram deixadas por três minutos em peneiras suspensas, à sombra, para o escorrimento da água excedente.

Foram determinados diariamente os valores de oxigênio dissolvido, temperatura, com oxímetro da YSI - 85, e pH, com peagômetro modelo pH-10 da Corning.

Para a avaliação dos resultados, os dados foram submetidos à análise de variância pelo teste F, sendo as médias dos tratamentos comparadas pelo teste de Tukey a $5 \%$, após serem transformadas em escala logarítmica seguindo a seguinte fórmula:

$$
\ln =(\text { efic. }+\alpha)
$$

em que

$$
\begin{aligned}
& \text { ln = logaritmo em base neperiana; } \\
& \text { efic. = eficiência de controle; e } \\
& \alpha=\text { coeficiente, com valor }=1
\end{aligned}
$$

\section{RESULTADOS E DISCUSSÃO}

Os valores médios de temperatura foram de $28,7 \pm 0,3{ }^{\circ} \mathrm{C}, 29,4 \pm 0,7{ }^{\circ} \mathrm{C}, 29,1 \pm 0,4{ }^{\circ} \mathrm{C}$ e $28,9 \pm 0,5^{\circ} \mathrm{C}$; os de oxigênio dissolvido, de $5,8 \pm 0,6 \mathrm{mg} \mathrm{L}^{-1}, 5,7 \pm 0,7 \mathrm{mg} \mathrm{L}^{-1}, 5,4 \pm 0,5 \mathrm{mg} \mathrm{L}^{-1}$ e $5,6 \pm 0,8 \mathrm{mg} \mathrm{L}^{-1}$ ); e os de $\mathrm{pH}$, de $8,35 \pm 0,53$, $8,21 \pm 0,43,8,17 \pm 0,29$ e $8,64 \pm 0,33$ nos experimentos 1 e 2 com período de três dias, nos experimentos de cinco e sete dias de duração, respectivamente. A baixa variação nos valores 
observados foi conseqüência da alta taxa de renovação diária dos tanques, fixada em 3,5 vezes o volume total.

Não se verificou variação significativa no crescimento dos peixes tanto em peso quanto em comprimento, o que já era esperado, devido ao curto espaço de tempo dos experimentos.

Observa-se que, quanto maior o período de duração do experimento, maior a eficiência de controle da massa verde total dos tratamentos (Tabela 1). Nos tanques em que foi oferecido $E$. densa $+C$. demersum o pacu se alimentou apenas de $E$. densa e não havia iniciado a predação sobre $C$. demersum no período experimental. Esse comportamento foi verificado nos experimentos de duração de três (Exp.1) e cinco dias (Tabela 1).

Verifica-se que o pacu teve potencial como agente de controle biológico de Egeria densa, E. najas e de Ceratophyllum demersum, uma vez que, em curto espaço de tempo, este peixe apresentou eficiência média de controle destas plantas daninhas aquáticas variando entre 28 e 100\%, ou seja, pode eliminar uma biomassa dessas plantas daninhas, com mesma quantidade de seu peso, em aproximadamente sete dias. Isso acontece devido ao fato de os peixes, que se alimentam de plantas, apesar de terem alta eficiência digestiva dos alimentos, necessitarem de alta ingestão de alimento, pois gastam alta quantidade de energia na sua assimilação (Welch, 1968).

Por meio da taxa de controle diário, que expressa o quanto o peixe controla por dia em relação ao seu peso em porcentagem, verificouse que o pacu controla entre 9,3 e $20,0 \%$ de seu peso vivo por dia de plantas daninhas aquáticas. Resultados semelhantes foram encontrados por Catarino et al. (1997) para a carpa-capim, com essa taxa variando entre 5 e $25 \%$ de seu peso por dia, para diferentes conjuntos de espécies de plantas daninhas.

Verificou-se que apenas o valor médio da eficiência de controle da biomassa total do tratamento E. densa $+E$. najas foi significativamente maior que o do tratamento $E$. densa + C. demersum, durante o experimento 1, com duração de três dias. Entretanto, observa-se que os valores do tratamento $E$. densa + $E$. najas durante os demais experimentos não diferiram significativamente do tratamento E. densa + C. demersum.

Tabela 1 - Valores médios da eficiência de controle do pacu (Piaractus mesopotamicus) sobre a massa verde total dos tratamentos contendo Egeria densa, E. najas e Ceratophyllum demersum, durante três, cinco e sete dias. Jaboticabal-SP, 2002

\begin{tabular}{|c|c|c|c|c|}
\hline \multicolumn{5}{|c|}{ Eficiência de Controle (\%) } \\
\hline Tratamento & 3 dias (exp.1) & 3 dias (exp.2) & 5 dias & 7 dias \\
\hline Egeria densa & $\begin{array}{c}47,9 \mathrm{AB} \\
(3,87)\end{array}$ & $\begin{array}{c}28,1 \\
(3,34)\end{array}$ & $\begin{array}{c}94,8 \\
(4,55)\end{array}$ & $\begin{array}{c}76,3 \\
(4,33)\end{array}$ \\
\hline Egeria najas & $\begin{array}{c}42,3 \mathrm{AB} \\
(3,74)\end{array}$ & $\begin{array}{c}35,8 \\
(3,57) \\
\end{array}$ & $\begin{array}{c}80,2 \\
(4,38)\end{array}$ & $\begin{array}{c}90,7 \\
(4,50)\end{array}$ \\
\hline Ceratophyllum demersum & $\begin{array}{c}38,6 \mathrm{AB} \\
(3,65)\end{array}$ & $\begin{array}{c}29,6 \\
(3,39)\end{array}$ & $\begin{array}{c}77,5 \\
(4,35) \\
\end{array}$ & $\begin{array}{c}83,9 \\
(4,42)\end{array}$ \\
\hline E. densa + E. najas & $\begin{array}{l}54,1 \mathrm{~A} \\
(3,99)\end{array}$ & $\begin{array}{c}42,7 \\
(3,75)\end{array}$ & $\begin{array}{c}88,1 \\
(4,47)\end{array}$ & $\begin{array}{c}100 \\
(4,61)\end{array}$ \\
\hline E. densa $+C$. demersum & $\begin{array}{l}32,0 \mathrm{~B} \\
(3,46)\end{array}$ & $\begin{array}{c}34,2 \\
(3,53)\end{array}$ & $\begin{array}{r}63,9 \\
(4,16) \\
\end{array}$ & $\begin{array}{c}76,6 \\
(4,33)\end{array}$ \\
\hline E. najas + C. demersum & $\begin{array}{c}46,5 \mathrm{AB} \\
(3,84)\end{array}$ & $\begin{array}{c}39,5 \\
(3,67)\end{array}$ & $\begin{array}{c}81,8 \\
(4,40)\end{array}$ & $\begin{array}{c}98,6 \\
(4,59)\end{array}$ \\
\hline E. densa + E. najas $+C$. demersum & $\begin{array}{c}49,1 \mathrm{AB} \\
(3,89)\end{array}$ & $\begin{array}{c}36,2 \\
(3,59)\end{array}$ & $\begin{array}{c}69,9 \\
(4,24)\end{array}$ & $\begin{array}{c}91,8 \\
(4,52)\end{array}$ \\
\hline DMS (em escala logarítmica) ${ }^{1 /}$ & 0,43 & 0,91 & 0,70 & 0,62 \\
\hline $\mathrm{CV}(\%)$ & 4,09 & 9,14 & 5,72 & 4,99 \\
\hline
\end{tabular}

Médias seguidas de mesma letra, na coluna, não diferem estatisticamente entre si pelo teste de Tukey $(<0,05)$; médias transformadas, escala logarítmica, entre parênteses; ${ }^{1 /}$ DMS - significativo a $5 \%$ de probabilidade. 
A eficiência de controle do pacu sobre $E$. densa e E. najas não apresentou redução significativa entre os tratamentos em todos os períodos de duração dos experimentos estudados (Tabelas 2 e 3). Fato contrário foi verificado para Ceratophyllum demersum, em que ocorreu redução significativa da eficiência de seu controle quando colocada juntamente com uma das espécies de Egeria, no experimento 1 com período de duração de três dias (Tabela 4), concordando com Duthu \& Kilgen (1975), Wiley et al. (1986) e Pine \& Anderson (1991), que observaram que esta planta foi menos controlada pela carpa-capim quando conjugada com outras plantas daninhas aquáticas.

Assim, a redução significativa da biomassa total de plantas no tratamento $E$. densa + C. demersum se deveu à redução do controle de C. demersum, ou seja, o pacu pode ter preferência ou ser seletivo pelas plantas do gênero Egeria a C. demersum. Isso também sugere que, nessas situações, E. densa e E. najas são controladas antes de $C$. demersum. Pine \& Anderson (1991) montaram um "ranking" de ordem de preferência da carpa-capim de 13 espécies de plantas daninhas aquáticas, em que $E$. densa e $C$. demersum foram classificadas em quarto e último lugares, respectivamente.

Tabela 2 - Valores médios da eficiência de controle de Egeria densa pela predação de pacu (Piaractus mesopotamicus), quando oferecidas isoladas ou em associação com Cerathophyllum demersum e E. najas, por períodos de três, cinco e sete dias. Jaboticabal-SP, 2002

\begin{tabular}{|c|c|c|c|c|}
\hline \multicolumn{5}{|c|}{ Eficiência de Controle (\%) } \\
\hline Tratamento & 3 dias (exp.1) & 3 dias (exp.2) & 5 dias & 7 dias \\
\hline Egeria densa & $\begin{array}{c}48,0 \\
(3,87)\end{array}$ & $\begin{array}{c}28,1 \\
(3,34)\end{array}$ & $\begin{array}{c}94,9 \\
(4,55)\end{array}$ & $\begin{array}{c}76,3 \\
(3,87)\end{array}$ \\
\hline E. densa + E. najas & $\begin{array}{c}45,0 \\
(3,81)\end{array}$ & $\begin{array}{l}29,31 \\
(3,37)\end{array}$ & $\begin{array}{c}93,1 \\
(4,53)\end{array}$ & $\begin{array}{c}100 \\
(4,61)\end{array}$ \\
\hline E. densa + C. demersum & $\begin{array}{c}55,8 \\
(4,02)\end{array}$ & $\begin{array}{c}48,4 \\
(3,88)\end{array}$ & $\begin{array}{c}96,3 \\
(4,56)\end{array}$ & $\begin{array}{c}100 \\
(4,61)\end{array}$ \\
\hline E. densa + E. najas $+C$. demersum & $\begin{array}{c}67,5 \\
(4,21)\end{array}$ & $\begin{array}{c}64,4 \\
(4,16)\end{array}$ & $\begin{array}{c}99,4 \\
(4,60)\end{array}$ & $\begin{array}{c}100 \\
(4,61)\end{array}$ \\
\hline DMS (em escala logarítmica) ${ }^{1 /}$ & 0,55 & 1,56 & 0,23 & 1,73 \\
\hline $\mathrm{CV}(\%)$ & 5,32 & 16,18 & 1,92 & 15,12 \\
\hline
\end{tabular}

Médias seguidas de mesma letra, na coluna, não diferem estatisticamente entre si pelo teste de Tukey $(<0,05)$; médias transformadas, escala logarítmica, entre parênteses; ${ }^{1 /}$ DMS - significativo a $5 \%$ de probabilidade.

Tabela 3 - Valores médios da eficiência de controle de Egeria najas pela predação de pacu (Piaractus mesopotamicus), quando oferecidas isoladas ou em associação com Cerathophyllum demersum e E. densa, durante três, cinco e sete dias. Jaboticabal-SP, 2002

\begin{tabular}{|c|c|c|c|c|}
\hline \multicolumn{5}{|c|}{ Eficiência de Controle (\%) } \\
\hline Tratamento & 3 dias (exp.1) & 3 dias (exp.2) & 5 dias & 7 dias \\
\hline Egeria densa & $\begin{array}{c}42,3 \\
(3,74)\end{array}$ & $\begin{array}{c}35,8 \\
(3,58) \\
\end{array}$ & $\begin{array}{c}93,4 \\
(4,54)\end{array}$ & $\begin{array}{c}90,8 \\
(4,51)\end{array}$ \\
\hline E. densa + E. najas & $\begin{array}{c}63,8 \\
(4,15)\end{array}$ & $\begin{array}{c}40,2 \\
(3,70)\end{array}$ & $\begin{array}{c}83,0 \\
(4,42)\end{array}$ & $\begin{array}{c}100 \\
(4,61)\end{array}$ \\
\hline E. najas $+C$. demersum & $\begin{array}{c}76,0 \\
(4,33)\end{array}$ & $\begin{array}{c}63,0 \\
(4,14)\end{array}$ & $\begin{array}{c}100 \\
(4,61)\end{array}$ & $\begin{array}{c}100 \\
(4,61)\end{array}$ \\
\hline E. densa + E. najas $+C$. demersum & $\begin{array}{c}53,5 \\
(3,99)\end{array}$ & $\begin{array}{c}22,1 \\
(3,10)\end{array}$ & $\begin{array}{c}78,4 \\
(4,36)\end{array}$ & $\begin{array}{c}100 \\
(4,61)\end{array}$ \\
\hline DMS (em escala logarítmica) ${ }^{1}$ & 0,64 & 2,91 & 0,47 & 0,23 \\
\hline $\mathrm{CV}(\%)$ & 6,07 & 11,25 & 4,02 & 1,93 \\
\hline
\end{tabular}

Médias seguidas de mesma letra, na coluna, não diferem estatisticamente entre si pelo teste de Tukey $(<0,05)$; médias transformadas, escala logarítmica, entre parênteses; ${ }^{\prime}$ DMS - significativo a $5 \%$ de probabilidade. 
Tabela 4 - Valores médios da eficiência de controle de Cerathophyllum demersum pela predação de pacu (Piaractus mesopotamicus), quando oferecidas isoladas ou em associação com Egeria najas e E. densa, durante três, cinco e sete dias. Jaboticabal-SP, 2002

\begin{tabular}{|c|c|c|c|c|}
\hline \multicolumn{5}{|c|}{ Eficiência de Controle (\%) } \\
\hline Tratamento & 3 dias (exp.1) & 3 dias (exp.2) & 5 dias & 7 dias \\
\hline Cerathophyllum demersum & $\begin{array}{l}37,9 \mathrm{~A} \\
(3,63)\end{array}$ & $\begin{array}{c}29,6 \\
(3,39) \\
\end{array}$ & $\begin{array}{c}77,5 \\
(4,35) \\
\end{array}$ & $\begin{array}{c}83,6 \\
(4,42)\end{array}$ \\
\hline E. densa + C. demersum & $\begin{array}{l}6,3 \mathrm{~B} \\
(1,84)\end{array}$ & $\begin{array}{c}8,4 \\
(2,13) \\
(2,13)\end{array}$ & $\begin{array}{c}29,0 \\
(3,37)\end{array}$ & $\begin{array}{c}57,4 \\
(2,97)\end{array}$ \\
\hline E. najas + C. demersum & $\begin{array}{l}8,4 \mathrm{~B} \\
(2,13)\end{array}$ & $\begin{array}{c}13,8 \\
(2,62)\end{array}$ & $\begin{array}{c}81,3 \\
(4,40)\end{array}$ & $\begin{array}{c}96,8 \\
(4,47)\end{array}$ \\
\hline E. densa + E. najas + C. demersum & $\begin{array}{c}15,0 \mathrm{AB} \\
(2,71)\end{array}$ & $\begin{array}{c}31,0 \\
(3,43)\end{array}$ & $\begin{array}{c}26,9 \\
(3,29)\end{array}$ & $\begin{array}{c}65,6 \\
(4,19)\end{array}$ \\
\hline DMS (em escala logarítmica $)^{1}$ & 1,33 & 2,47 & 1,10 & 1,74 \\
\hline $\mathrm{CV}(\%)$ & 41,23 & 32,65 & 46,27 & 33,33 \\
\hline
\end{tabular}

Médias seguidas de mesma letra, na coluna, não diferem estatisticamente entre si pelo teste de Tukey $(<0,05)$; médias transformadas, escala logarítmica, entre parênteses; ${ }^{1 /}$ DMS - significativo a $5 \%$ de probabilidade.

Segundo Wiley et al. (1986), os peixes preferem as plantas que podem consumir mais rapidamente, otimizando o ganho de energia, uma vez que esses mesmos autores verificaram que não existe correlação entre a preferência de consumo da carpa-capim e o conteúdo de proteína e energia das plantas daninhas aquáticas.

A não-existência de diferença significativa da eficiência de controle entre os tratamentos onde as plantas foram colocadas individualmente, principalmente $C$. demersum, bem como o aumento da sua eficiência com o aumento do período de duração dos experimentos, são explicados pelo fato de que, na ausência de plantas preferidas, os peixes também controlam as menos preferidas (Fowler \& Robson, 1978; Van Dyke et al., 1984) e também pelo fato de que em temperaturas altas, como as ocorridas nos experimentos, a seletividade dos peixes diminui, já que a demanda energética do peixe é maior (Edwards, 1974).

Os resultados são importantes, considerando que o repovoamento de peixes nativos em reservatórios de hidrelétricas é uma condicionante de funcionamento requerida pelos órgãos de defesa do meio ambiente. O repovoamento do pacu dirigido para reservatórios com grande incidência de plantas daninhas submersas pode ser uma prática de bons resultados no manejo integrado desse tipo de vegetação.

\section{AGRADECIMENTOS}

Ao Prof. Dr. José Carlos Barbosa, do Depto. de Ciências Exatas da Faculdade de Ciências Agrárias e Veterinárias, UNESP, campus de Jaboticabal, e ao Prof. Dr. Dalton José Carneiro, do Centro de Aqüicultura, UNESP, campus de Jaboticabal.

\section{LITERATURA CITADA}

BARKO, J. W.; ADAMS, M. S.; CLLESCERI, N. L. Environmental factors and their consideration in the management of submersed aquatic vegetation: a review. J. Aquatic Plant Manag., v. 24, p. 1-10, 1986.

BLACKBURN, R.D., SUTTON, D.L., TAYLOR, T. Biological control of aquatic weeds. J. Irrig. Drainase, v. 97, n. 3, p. 421-32, 1971.

BOYD, C. E. The limnological role of aquatic macrophytes and their relationship to reservoir management. Am. Fish. Soc. Spec. Publ., v. 8, p. 153-166, 1971.

BUA-NGAM, T.; MERCADO, B. L. Competition of water lettuce (Pistia stratiotes) with rice and commonly associated weed species. Philipp. Agric., v. 60, p. 22-30, 1976.

CASTAGNOLLI, N. Criação de peixes de água doce. Jaboticabal: FUNEP, 1992, 189 p.

CATARINO, L. F.; FERREIRA, M. T.; MOREIRA, I. S. Preferences of grass carp for macrophytes in iberian drainage channels. J. Aquatic Plant Manag. v. 36, p. 7983, 1997. 
DA SILVA, A. J. Aspectos da alimentação do pacu adulto, Colossoma metrei (Berge, 1895) (PISCES, CHARACIDAEA), no Pantanal do Mato Grosso. 1982, 118 f. Dissertação (Mestrado em Ciências Biológicas Zoologia) - Universidade Federal Rural do Rio de Janeiro, Rio de Janeiro, 1982.

DUTHU, G. S.; KILGEN, R. H. Aquarium studies on the selectivity of 16 aquatic plants as food by fingerling hybrids of cross between Ctenopharyngodon idella and Cyprinus carpio. J. Fish Biol., v.7, p.203-208, 1975.

EDWARDS, D. J. Weeds preference and growth of young grass carp in New Zealand. New Zealand J. Marine Freshw. Res., v. 8, n. 2, p. 341-350, 1974.

FOLWER, M. C.; ROBSON, T. O. The effects of the food preferences and stocking rates of grass carp (Ctenopharyngodon idella Val.) on mixed plants communities. Aquatic Bot., v. 5, p. 261-276, 1978.

GRAVENA, S.; LARA, F. M. Comparação de métodos para avaliação da eficiência de defensivos no controle de Perileucoptera coffeela (Guer.- menev., 1842). Científica, v. 4, n. 2, p. 97-100, 1976.

HALLER, W. T. Hydrilla: a new and rapidly spreading aquatic weed problem. Gainesville: University of Florida, 1976, 13 p. (Circular, 5-245).

NACHTIGAL, G. L. Desempenho de agente de controle biológico microbiano de Egeria najas L. e de Egeria densa L. 2000, 169 f. Tese (Doutorado em Produção Vegetal) Universidade Estadual de São Paulo, Jaboticabal, 2000.
PINE, R. T.; ANDERSON, W. J. Plant preferences of triploid grass carp. J. Aquatic Plant Manag., v. 29, p. 8082, 1991.

RIMER, D. N. Introduction to freshwater vegetation: London: AVI Plublishing, 1984. 207p.

SAEED, M. O.; ZIEBELL, C. D. Effects of dietary nonprefered aquatic plants on the growth of redbelly tilapia (Tilapia zilli). Progr. Fish-Cult.., v. 48, p. 110-112, 1986.

STANLEY, J. G. Nitrogen and phosphorus balance of grass carp Ctenopharyngodon idella, fed elodea, Egeria densa. Trans. Am. Fish Soc., n. 3, p. 587-592, 1974.

SUTTON, D. L., VANDIVER, R. V. V. Grass carp: A fish for biological management of hydrilla and other aquatic weeds in Florida. Gainesville, 1986. 10 p. (Bulletim of Florida Agricultural Experiment Stations, n. 867)

VAN DIKE, M. J.; LESLIE, A. J.; NALL, L. E. The effects of the grass carp on the aquatic macrophytes of four Florida lakes. J. Aquatic Plant Manag., v. 22, p. 87-95, 1984.

WELCH, H. E. Relationsihps between assimilation efficiencies and growth efficiencies for aquatic consumers. Ecology, v. 49, p. 755-759, 1968.

WILEY, M. J.; PESTICELLI, S. M.; WIKE, L. D. The relationship between feeding preferences and consumption rates in grass carp and grass carp $\mathrm{x}$ bighead carp hybrids. J. Fish Biol., v. 29, p. 507-514, 1986. 